\title{
AEGIS AT CERN: MEASURING ANTIHYDROGEN FALL
}

\author{
MARCO G. GIAMMARCHI \\ Istituto Nazionale di Fisica Nucleare \\ Via Celoria 16, Milano 20133, Italy \\ E-mail: marco.giammarchi@mi.infn.it \\ on behalf of the AEGIS Collaboration*
}

\begin{abstract}
The main goal of the AEGIS experiment at the CERN Antiproton Decelerator is the test of fundamental laws such as the Weak Equivalence Principle (WEP) and CPT symmetry. In the first phase of AEGIS, a beam of antihydrogen will be formed whose fall in the gravitational field is measured in a Moiré deflectometer; this will constitute the first test of the WEP with antimatter.
\end{abstract}

\section{Introduction}

The goal of AEGIS (Antimatter Experiment: Gravity, Interferometry, Spectroscopy) ${ }^{1}$ under construction at CERN, is the study of fundamental physics with antimatter, namely the investigation of the Weak Equivalence Principle (WEP) and CPT symmetry. This constitutes a test of the foundations of General Relativity and quantum field theory. ${ }^{2,3}$

During the first phase of the experiment, the production of a antihydrogen $(\overline{\mathrm{H}})$ beam is foreseen to allow tests of the WEP by producing antihydrogen through the charge-exchange reaction $(\mathrm{Ps})^{*} \bar{p} \rightarrow \overline{\mathrm{H}}^{*} e^{-}$and forming a $\overline{\mathrm{H}}$ beam whose fall in the gravity field is measured with a Moiré deflectometer.

*A.S. Belov, G. Bonomi, I. Boscolo, R.S. Brusa, V.M. Byakov, L. Cabaret, A. Calloni, C. Canali, C. Carraro, F. Castelli, S. Cialdi, D. Comparat, G. Consolati, N. Djourelov, M. Doser, G. Drobychev, A. Dudarev, A. Dupasquier, D. Fabris, R. Ferragut, G. Ferrari, A. Fischer, P. Folegati, A. Fontana, M. Lunardon, M.G. Giammarchi, S.N. Gninenko, R. Heyne, S.D. Hogan, L.W. Jorgensen, A. Kelleberbauer, D. Krasnicky, V. Lagomarsino, G. Manuzio, S. Mariazzi, V.A. Matveev, F. Merkt, S. Moretto, C. Morhard, G. Nebbia, P. Nedelec, M.K. Oberthaler, D. Perini, S. Pesente, V. Petracek, M. Prevedelli, I.Y. AlQaradawi, F. Quasso, C. Riccardi, O. Rohne, A. Rotondi, M. Sacerdoti, H. Sandaker, D. Sillou, S.V. Stepanov, H.H. Stroke, G. Testera, D. Trezzi, A.V. Turbabin, G. Viesti, F. Villa, H. Walters, U. Warring, S. Zavatarelli, A. Zenoni, D.S. Zvezhinskij. 
In a second phase of the experiment, $\overline{\mathrm{H}}$ will be laser-cooled and confined, to perform higher precision $g$ measurements and CPT tests.

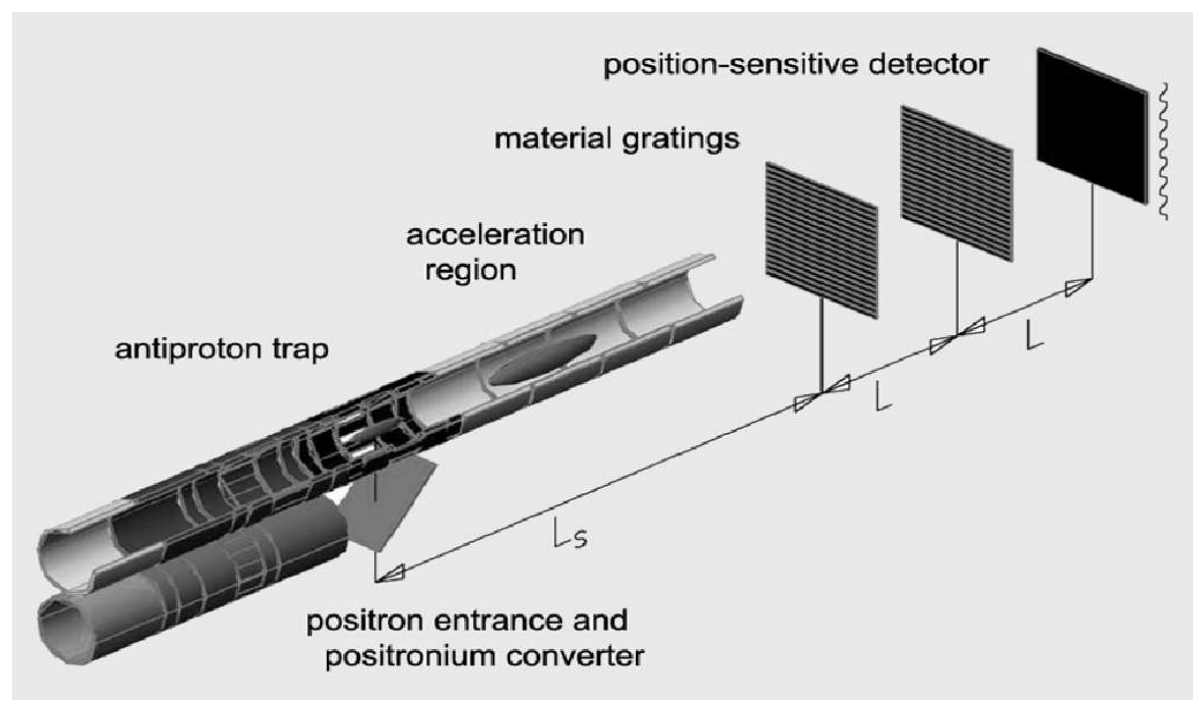

Fig. 1. Sketch of the central part of AEGIS. Two parallel Penning-Malmberg traps $(r=8 \mathrm{~mm})$ manipulate $\bar{p}$ and $e^{+}$to form and accelerate $\overline{\mathrm{H}}$. They will be mounted inside a $100 \mathrm{mK}$ cryostat in a $1 \mathrm{~T}$ magnetic field. The upper trap is devoted to antiprotons. Cold $\bar{p}$ wait for Ps in the black region. The lower trap is devoted to positrons; they will be sent on to the converter to produce Ps. Laser pulses will excite the Ps to Rydberg states to form $\bar{p}$ in the black region. The acceleration region shows the bunch of antihydrogen after the Stark acceleration. The two material gratings $\left(L_{s}=50 \mathrm{~cm}, L=30 \mathrm{~cm}\right.$, transverse dimensions: $20 \times 20 \mathrm{~cm}^{2}$ ) and the detector are used for the $g$ measurement.

The best sensitivity for WEP tests made on matter systems come from rotating torsion balances ${ }^{4}$ and from the Moon and Earth accelerations; ${ }^{5}$ they are both in the range of $\sim 10^{-13}$. On the other hand, there have been no direct measurements of the gravitational acceleration of antimatter.

\section{The production of the antihydrogen beam}

The measurement of $g$ is performed using the gravitational fall of a beam of antihydrogen, whose production involves the following steps.

- Production of positrons in a Surko-type source and accumulator

- Accumulation and cooling of the antiprotons

- Production of Ps by positron-bombardment of a converter

- Laser excitation of the Ps to a $n \simeq 20-25$ Rydberg state 
- Production of $\overline{\mathrm{H}}$ by means of the reaction $(\mathrm{Ps})^{*} \bar{p} \rightarrow \overline{\mathrm{H}}^{*} e^{-}$

- Formation of a $\overline{\mathrm{H}}$ beam by Stark acceleration

- Measurement of $g$ in a Moiré deflectometer

Figure 1 shows a schematics of the core of the apparatus, whose antiproton (Penning-Malberg) trap is immersed in a $1 \mathrm{~T}$ magnetic field for charged particle confinement.

After antiproton cooling (requiring about $300 \mathrm{~s}$ ), the positron bunch is fired at the converter to produce Ps for the charge exchange reaction. The production process is therefore a pulsed production triggered by the $e^{+}$ hitting the converter. The produced Ps gets laser excited before intersecting the $\bar{p}$ cloud. The $\overline{\mathrm{H}}$ production process takes about a microsecond; after that, the antiatoms are accelerated towards the deflectometer by means of a Stark acceleration technique. The $\bar{p}$ beam fall will be measured with a Moiré deflectometer equipped with a position sensitive microstrip detector.

Antiprotons are coming from the CERN Antiproton Decelerator (AD), delivering about $3 \times 10^{7}$ particles every $100 \mathrm{~s}$ at $6 \mathrm{MeV}$. After an energy degrader, antiprotons are caught in a $3 \mathrm{~T}$ magnetic field region (not shown in Fig. 1) at a temperature of $4 \mathrm{~K}$ and further cooled down to $100 \mathrm{mK}$ (a velocity of $\sim 50 \mathrm{~m} / \mathrm{s}$ ). This low temperature is achieved by resistive cooling or sympathetic cooling with laser cooled osmium ions. ${ }^{6}$

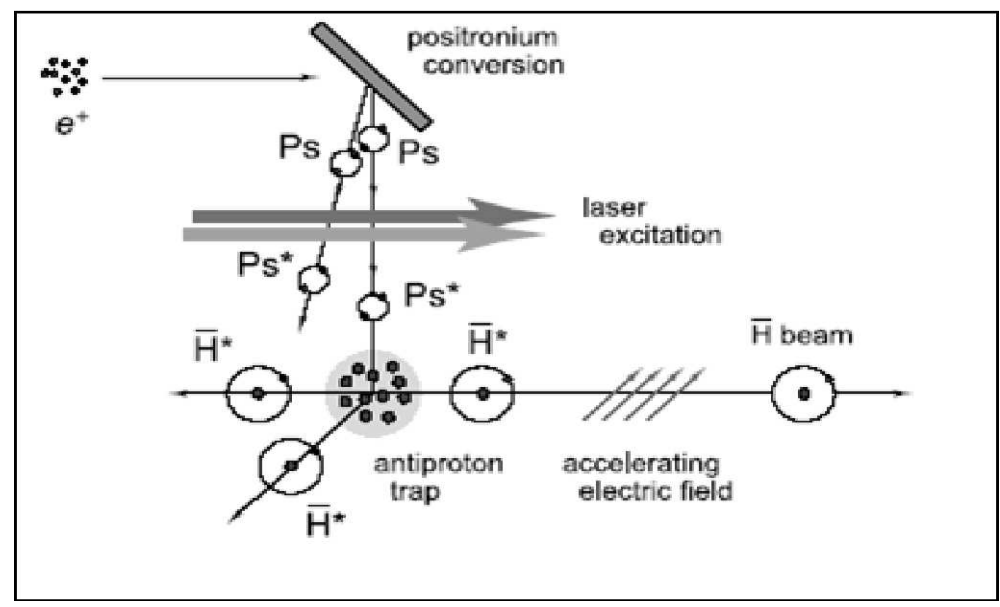

Fig. 2. Schematics of the AEGIS antihydrogen production and acceleration process. The positron bunch lasts about $20 \mathrm{~ns}$ while the laser pulse is $10 \mathrm{~ns}$ long. 


\subsection{Positronium formation and excitation}

Positronium will be formed by an $e^{+}$bunch hitting a converter. Positrons will be produced by a Surko-type accumulator delivering a $20 \mathrm{~ns}$ bunch of about $10^{8}$ particles with a few keV energy.

After Ps has been formed in the bulk of the converter material, ortho-Ps can be reemitted out of the target (Fig. 2). experiments have shown that in suitable converter materials, reemitted Ps in the amount of about $50 \%$ of the impinging $e^{+}$can be obtained..$^{7-9}$

The excitation of Ps to Rydberg states is necessary since the cross section of the reaction $(\mathrm{Ps})^{*} \bar{p} \rightarrow \overline{\mathrm{H}}^{*} e^{-}$has a strong dependence on the principal quantum number of the Ps, $\sigma \sim a_{0} n^{4}$ ( $a_{0}$ being the Bohr radius). Positronium will be laser excited to a high- $n$ (Rydberg) state using a $1 \rightarrow 3 \rightarrow n$ excitation process, where $n=20-25 .{ }^{10}$

\subsection{Antihydrogen formation and acceleration}

Taking into account the production cross section, the geometry of the system and the number of $\bar{p}$ and excited Ps atoms, about 500 antihydrogen atoms will be formed per positron shot on the converter. Since this process follows the accumulation of $e^{+}$in the positron accumulator and the accumulation and cooling of $\bar{p}$ in the catching region (300 s), the $\overline{\mathrm{H}}$ averaged production rate will be of a few Hz. The low temperature of the antiproton cloud allows the production of antihydrogen with an energy of $100 \mathrm{mK}$, or a thermal speed of $50 \mathrm{~m} / \mathrm{s}$ (similar to the antiproton energy).

The produced $\overline{\mathrm{H}}$ will be Stark-accelerated along the beam axis using the technique recently demonstrated for hydrogen. ${ }^{11}$ The $\overline{\mathrm{H}}$ atoms will arrive at the beginning of the deflectometer with an axial velocity of $200-500 \mathrm{~m} / \mathrm{s}$ and a radial velocity spread of $\sim 50 \mathrm{~m} / \mathrm{s}$.

\section{The gravity measurement}

Measuring $g$ with a flight path of $60 \mathrm{~cm}$ and a velocity $v=500 \mathrm{~m} / \mathrm{s}$ involves measuring a displacement of $\simeq 20 \mu \mathrm{m}$ against an $8 \mathrm{~mm}$ beam spot. This will be done with a classical Moiré deflectometer; the device ${ }^{12}$ consists of three equally spaced and parallel material gratings (Fig. 3). The last plane will be a position-sensitive microstrip detector to register the time and impact point of $\overline{\mathrm{H}}$ atoms.

As the atomic beam passes through the gratings the first two planes select specific propagation directions creating on the third plane a density modulation repeating itself at positions that are integer multiples of the 
distance between the first two gratings. This technique, originally proposed in Ref. 13, can be effectively applied to the case of inertial sensing (and gravity measurements) as discussed in Ref. 12 .

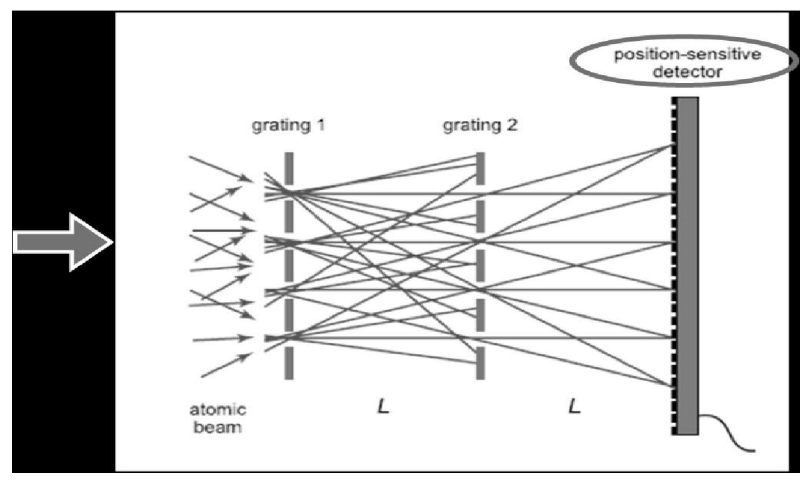

Fig. 3. Principle of the Moirè deflectometer and the detector for AEGIS. $L$ is $30 \mathrm{~cm}$.

The modulation intensity pattern will be shifted by a quantity $\delta$ that depends on the transit time $T$, the period $a$ of the grating and $g: \delta=$ $g T^{2} / a$. The gravity constant $g$ will be measured by fitting this distribution. Taking into account several possible sources of errors, a final $1 \%$ resolution on $g$ can be achieved by launching $10^{5}$ antihydrogen atoms towards the deflectometer. This amounts to a few months of data taking.

\section{References}

1. A. Kellerbauer et al., Nucl. Instr. Meth. B 266, 351 (2008).

2. N.E. Mavromatos, in A. Hirtl, J. Marton, E. Widmann, and J. Zmeskal, eds., International Conference on Exotic Atoms and Related Topics, Austrian Academy of Sciences, Vienna, 2006.

3. Data Tables for Lorentz and CPT Violation, 2010 edition, V.A. Kostelecký and N. Russell, arXiv:0801.0287v3.

4. S. Schlamminger et al., Phys. Rev. Lett. 100, 041101 (2008).

5. J.G. Will et al., Phys. Rev. Lett. 93, 261101 (2004).

6. U. Warring et al., Phys. Rev. Lett. 102, 043001 (2009).

7. L. Lizkay et al., Appl. Phys. Lett. 92, 063114 (2008).

8. S. Mariazzi et al., Phys. Rev. B 68, 085428 (2008).

9. R. Ferragut et al., submitted to Can. J. Phys.

10. F. Castelli et al., Phys. Rev. A 78, 052512 (2008).

11. E. Vliegen et al., Phys. Rev. A 76, 023405 (2007).

12. M.K. Oberthaler et al., Phys. Rev. A 54, 3165 (1996).

13. O. Kafri, Opt. Lett. 5, 555 (1980). 